\title{
Thermodynamical Fluxes for the Modeling of Cardiac Mitochondrial Calcium Handling
}

\author{
Bachar Tarraf ${ }^{1,2,3}$, Michael Leguèbe ${ }^{1,2,3}$, Yves Coudière ${ }^{4,3,1,2}$, Philippe Diolez ${ }^{2,4,5}$ \\ ${ }^{1}$ Inria Bordeaux-Sud-Ouest team CARMEN, Talence, France \\ ${ }^{2}$ IHU Liryc, Fondation Bordeaux Université, Pessac, France \\ ${ }^{3}$ Institut de Mathématiques de Bordeaux UMR CNRS 5251, Talence, France \\ ${ }^{4}$ Université de Bordeaux, Bordeaux, France \\ ${ }^{5}$ INSERM, Centre de recherche Cardio-Thoracique de Bordeaux, U1045, Bordeaux, France
}

\begin{abstract}
It is known that mitochondria play a crucial role in the handling of calcium by cardiac cells during the excitationcontraction cycle. However, the precise characterization of this role is still under debate.

With this intent, a collection of mathematical models have been developed, but they generally show a level of complexity that is not compatible with inverse problem techniques required for calibration with experimental data. Their large number of equations and parameters can also lead to transcription mistakes that can be found in the literature. In this paper we apply a similar methodology as Bertram et al.in [1] to propose a simple model that is derived from the base equations that constitute the origin of most mitochondrial models [2].

Our model describes the main features of the mitochondrial activity with 6 equations and $\sim 30$ parameters, which we will eventually reduce in a forthcoming sensitivity analysis.
\end{abstract}

\section{Introduction}

Calcium $\left(\mathrm{Ca}^{2+}\right)$ handling is crucial in cardiac excitationcontraction coupling. Several cellular mechanisms regulate the cytoplasmic $\mathrm{Ca}^{2+}$ concentration during the cardiac cycle, in particular the sarcoplasmic reticulum, myofibrils and mitochondria. It is known that arrhythmias may occur due to dysfunctions in $\mathrm{Ca}^{2+}$ regulation. However, the exact role of mitochondria in $\mathrm{Ca}^{2+}$ handling during the cell contraction and relaxation is still an open question [3-5]. In this paper we present a mathematical model of $\mathrm{Ca}^{2+}$ handling by mitochondria. It may be coupled to a cellular contraction model. The model is derived in collaboration with biologists, and written in a way that will facilitate its forthcoming calibration with respect to experimental data: the number of state equations and parameters is kept as low as possible, still being able to capture the main dynamics of interest.

There exists abundant literature about mitochondrial models. However, most of it show a level of complexity that makes parameter fitting extremely difficult, if not mathematically impossible. These models are derived following a cumulative approach, including additional mechanics and biological pathways to previous models, which leads to dozens of state variables and hundreds of parameters. Also, as the complexity grows, the risk of transcription mistakes in the equations increases, as reported by Saa and Siquiera [6].

A more phenomenological approach was proposed by Bertram et al. [1] by selecting core elements of the mitochondrial activity and simplifying the relations between them. It was based on the equations of Cortassa et al. [7]. However, some hypotheses that are not relevant to our context were made, and some mistakes were also committed [6].

We followed a similar simplification approach to derive our model, using the method in Magnus and Keizer [2], on which the equations of Cortassa et al.are based, and being meticulous in our simplification process.

The next section of this paper introduces the equations which constitute the model. These equations contain the expressions of ionic species fluxes across mitochondrial membrane, that are detailed in the third section. The last section contains some remarks about our model, and concludes with future work.

\section{Mathematical model of mitochondria}

The complexity of mitochondrial models derived from the Magnus-Keizer (MK) model comes generally from the quantity of biophysical phenomena that are described. Here we derive a model that selects key mechanisms of the mitochondria in the context of the cellular contraction 


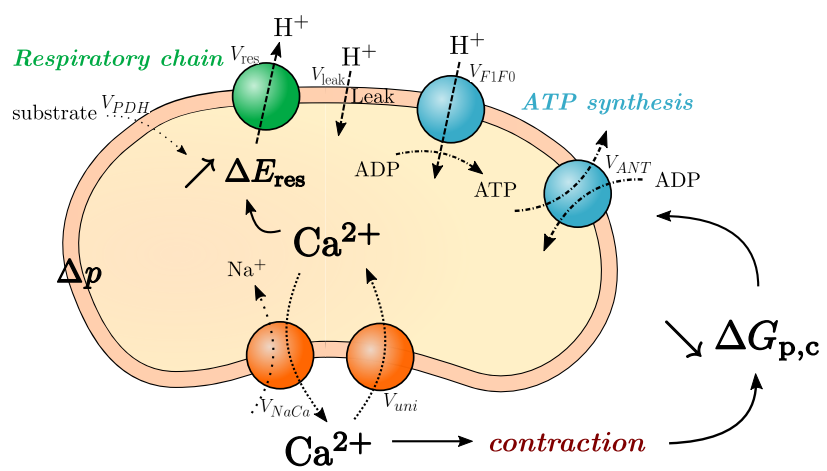

Figure 1. Reaction and fluxes described by the model. State variables are indicated by bold font.

induced by calcium. Considering that mitochondria are placed in a medium with over-abundance of oxygen and substrate, our equations will focus on NADH oxidation, ATP-production and exchange with the cytoplasmic compartment, calcium handling through $\mathrm{Ca}$ uniporter and $\mathrm{Na}-$ $\mathrm{Ca}$ exchanger, and finally proton leakage across the membrane. Figure 1 regroups schematically the modeled elements of the mitochondrial cycle.

Models from the literature monitor intra and extra mitochondrial chemical concentrations. Here, we choose to describe the mitochondrial behavior through thermodynamic variables (actually related to electrochemical force induced by chemical gradients of concentration). These variables are expressed relatively to their values when the mitochondria is at state 4 , so that their sign characterizes the activity of mitochondria.

We present now these thermodynamic variables, how they are coupled, and their dynamics. The expression of the fluxes in these equations will be stated in next section.

NADH redox potential. NADH is used by respiratory complex to generate a proton gradient across the mitochondrial membrane, yielding $\mathrm{NAD}^{+}$. The availability of NADH in the matrix is expressed through the redox potential $\Delta E_{\text {resp }}($ in $\mathrm{mV}$ ) defined as

$$
\Delta E_{\mathrm{resp}}=\frac{R T}{F} \ln \left(K_{\mathrm{resp}} \sqrt{\frac{[\mathrm{NADH}]_{\mathrm{m}}}{\left[\mathrm{NAD}^{+}\right]_{\mathrm{m}}}}\right),
$$

where $R, T$ and $F$ are the gas constant, temperature and Faraday's constant, and $K_{\text {resp }}$ is the redox equilibrium constant. The total concentration of nicotinamide adenine nucleotides is supposed constant:

$$
\left[\mathrm{NAD}^{+}\right]_{\mathrm{m}}+[\mathrm{NADH}]_{\mathrm{m}}=[\mathrm{NAD}]_{\mathrm{tot}} .
$$

$\Delta E_{\text {resp }}$ decreases due to the electron transport chain (ETC) which oxidizes NADH into $\mathrm{NAD}^{+}$, and increases with the production of NADH, which is modeled as in [1] by a single compartment regrouping glycolysis and Krebs cycle. This leads to the following ODE :

$$
\frac{\mathrm{d}}{\mathrm{dt}} \Delta E_{\mathrm{resp}}=\varphi_{1}\left(\Delta E_{\mathrm{resp}}\right)\left(V_{\mathrm{PDH}}-V_{\text {resp }}\right) .
$$

where $\varphi_{1}$ comes from the change of variable in the ODE originally expressed for $[\mathrm{NADH}]_{\mathrm{m}}$, and includes the parameters $K_{\text {resp }}$ and $[\mathrm{NAD}]_{\text {tot }}$

Gibbs Free Energy. Together with the regulation by calcium, perturbation of the energetic balance between ATP and ADP in the cytoplasm is an activation mechanism of the mitochondria. We choose to use it as a variable of our model, and it is defined (in units of $\mathrm{mV}$ ) as

$$
\Delta G_{\mathrm{p}}=\frac{R T}{F} \ln \left(K_{\mathrm{F} 1} \frac{[\mathrm{ATP}]}{[\mathrm{ADP}][\mathrm{Pi}]}\right),
$$

for both the cytoplasmic and mitochondrial compartments. The variables will be named $\Delta G_{\mathrm{p}, \mathrm{c}}$ and $\Delta G_{\mathrm{p}, \mathrm{m}}$ respectively. We do not assume that the ATP/ADP ratio is the same in the matrix and in the cytoplasm, since it is not relevant in our context.

Again, we consider the total quantity of adenine nucleotides to be constant

$$
[\mathrm{ADP}]_{\mathrm{m}}+[\mathrm{ATP}]_{\mathrm{m}}=[\mathrm{A}]_{\mathrm{tot}} .
$$

In the matrix, $\Delta G_{\mathrm{p}, \mathrm{m}}$ is modified by the activity of ATPsynthase and the ATP/ADP translocator, which also acts on $\Delta G_{\mathrm{p}, \mathrm{c}}$. We have:

$$
\begin{aligned}
& \frac{\mathrm{d}}{\mathrm{dt}} \Delta G_{\mathrm{p}, \mathrm{m}}=\varphi_{2}\left(\Delta G_{\mathrm{p}, \mathrm{m}}\right)\left(V_{\mathrm{F} 1 \mathrm{~F} 0}-V_{\mathrm{ANT}}\right), \\
& \frac{\mathrm{d}}{\mathrm{dt}} \Delta G_{\mathrm{p}, \mathrm{c}}=\varphi_{3}\left(\Delta G_{\mathrm{p}, \mathrm{m}}, \Delta G_{\mathrm{p}, \mathrm{c}}\right) V_{\mathrm{ANT}}+\dot{g}_{\mathrm{ext}}(t)
\end{aligned}
$$

where the ADP fluxes $V_{\mathrm{ANT}}$ and $V_{\mathrm{F} 1 \mathrm{~F} 0}$ are derived by simplification of the MK model. The function $\varphi_{2}$ takes into account our change of variables from $[\mathrm{ATP}]_{\mathrm{m}}$ to $\Delta G_{\mathrm{p}, \mathrm{m}}$. The "external" factors acting on $\Delta G_{\mathrm{p}, \mathrm{c}}$, such as myofibril contraction or experimental additions of ATP/ADP, are represented by the source term $\dot{g}_{\text {ext }}$.

Proton-motive force. We choose to use in our model the proton-motive force $\Delta p$ which has units of $\mathrm{mV}$, and is the combination of the electric potential across the inner membrane $\Delta \psi$ and the proton gradient $\Delta \mathrm{pH}$ (Eq. 3.33 of [8]). Hence, all flux terms contribute in a similar way to $\Delta p$, whether they produce/consume protons (pumps) or other electric charges (Na-Ca exchanger, leaks). The proton-motive force is defined as

$$
\Delta p=\Delta \psi-\ln (10) \frac{R T}{F} \Delta \mathrm{pH} .
$$


With the exception of NADH production, all reactions of our model act on $\Delta p$, and then we have

$C_{\mathrm{m}} \frac{\mathrm{d}}{\mathrm{dt}} \Delta p=12 V_{\text {resp }}-3 V_{\mathrm{F} 1 \mathrm{~F} 0}-V_{\mathrm{ANT}}-V_{\text {leak }}-2 V_{\mathrm{uni}}$,

where $C_{\mathrm{m}}$ is the membrane capacitance. The coefficients 12,3 and 2 come from the stoichiometry of the respiratory chain and the ATP-synthase in terms of protons, and from the two charges of $\mathrm{Ca}^{2+}$ going through uniporter. There is no charge flux due to the Na-Ca exchanger, that is considered electro-neutral.

Calcium. We follow the concentration of $\mathrm{Ca}^{2+}$ in the mitochondria because it is the quantity of main interest for us. $\mathrm{Ca}^{2+}$ enters and exits the matrix by the $\mathrm{Ca}^{2+}$ uniporter and the $\mathrm{Na}-\mathrm{Ca}$ exchanger respectively, so that we have

$$
\frac{\mathrm{d}}{\mathrm{dt}}\left[\mathrm{Ca}^{2+}\right]_{\mathrm{m}}=f\left(V_{\mathrm{uni}}-V_{\mathrm{NaCa}}\right),
$$

where $f$ is the fraction of free $\mathrm{Ca}^{2+}$ in the matrix. The cytoplasmic calcium concentration also follows an ODE, which may mimic a cellular calcium cycle, or any experimental condition, as the source term $\dot{c}$ of the equation:

$$
\frac{\mathrm{d}}{\mathrm{dt}}\left[\mathrm{Ca}^{2+}\right]_{\mathrm{c}}=-\gamma \frac{\mathrm{d}}{\mathrm{dt}}\left[\mathrm{Ca}^{2+}\right]_{\mathrm{m}}+\dot{c}(t) .
$$

where $\gamma$ is the fraction of free cytoplasmic calcium.

\section{Derivation of flux expressions}

In this section we give the definitions of the fluxes used in the differential system of Eqs. 3, 6, 7, 9, 10, and 11. We only detail the derivation of the proton flux of the respiratory complex, since all other fluxes are derived using similar strategy.

In the literature, the expression of $V_{\text {resp }}\left([\mathrm{NADH}]_{\mathrm{m}}, \Delta \psi\right)$ is generally derived from Eq. 6 in [2]. However, information on how this formula was derived are partial, and it is difficult to determine the modeling process behind all the terms. To better understand this, we used the method proposed by Hill [9] which is also the origin of Magnus and Keizer equations.

The concept is to relate an exorgenic reaction to an outward proton flux across a macromolecular membranebound complex. In our case, NADH oxidation is involved in complex I, III, and IV of the respiratory chain, where protons are pumped. The resulting redox reaction is:

$$
\frac{1}{2} \mathrm{NADH}+\frac{1}{2} \mathrm{H}^{+}+\frac{1}{4} \mathrm{O}_{2}=\frac{1}{2} \mathrm{NAD}^{+}+\frac{1}{2} \mathrm{H}_{2} \mathrm{O}
$$

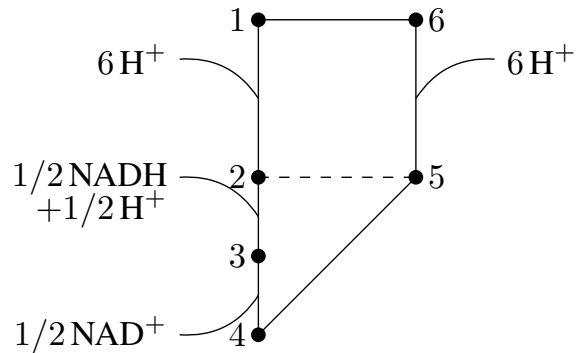

Figure 2. Altman-King-Hill diagram of the respiratory chain. Further details appear in the text.

The $e^{-} / \mathrm{H}^{+}$stoichiometry was adapted from the work of Magnus et al.[2]. For each electron transferred through the ETC, 6 protons will be pumped ( 2 per site). In diagram 2, the left and the right sides represent matrix and cytoplasm respectively. Each node is a state of the enzymatic complex, and each edge is a possible transition between those states. The arcs associated with the diagram represent the binding or unbinding of molecules to the enzymatic complex. The dashed edge between states 2 and 5 represent the proton slip or the reaction slip which may occur during the whole cycle. However, for simplicity, we suppose that these mechanisms are negligible before the whole leakage occurring across the membrane outside the respiratory complex. Thus, we removed this edge from the diagram, which simplifies considerably the expression of our flux. The respiration driven flux is written using rate constants associated to each edge of the diagram, and can be either constants or functions of the molecules concentrations or the membrane potential:

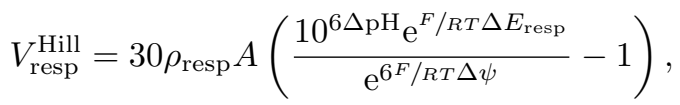

where $A$ is a function of $\Delta E_{\text {resp }}$ and $\Delta \psi$ that comes from the Altman-King-Hill analysis, and includes 14 parameters. This definition is used as a reference against which we fit a function with less parameters. We chose the following function to fit the original flux, which is close to a product of sigmoid profiles:

$$
\begin{gathered}
V_{\text {resp }}=\sigma\left(\Delta E_{\text {resp }}, 0, \sigma\left(\Delta \psi, p_{0}, 0, p_{1}, p_{2}\right),\right. \\
\left.p_{3}, \sigma\left(\Delta \psi, p_{4}, p_{5}, p_{1}, p_{2}\right)\right)
\end{gathered}
$$

where

$\sigma\left(x, s_{1}, s_{2}, k, x_{0}\right)=s_{1}+\frac{s_{2}-s_{1}}{2}\left(1+\tanh \left(k\left(x-x_{0}\right)\right)\right)$

is a sigmoid function transitioning from $s_{1}$ to $s_{2}$ around $x=x_{0}$ with slope $k$. $V_{\text {resp }}^{\text {Fit }}$ was chosen after performing bidirectional fits along $\Delta E_{\text {resp }}$ and $\Delta p$, already identifying parameters that remain constant. The fitted function 


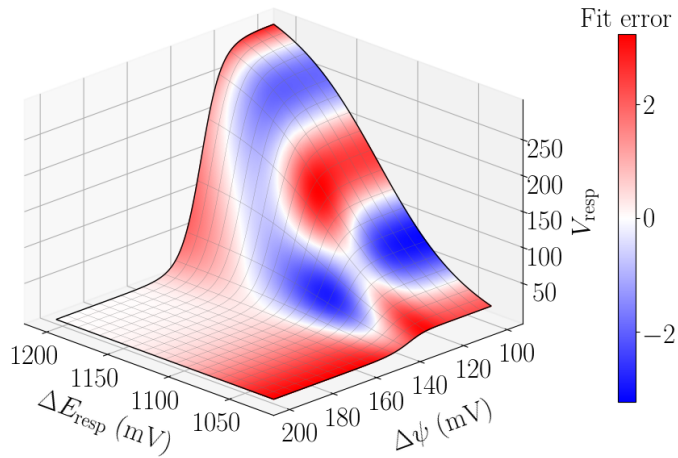

Figure 3. Fit of flux $V_{\text {resp }}$, defined in Eq. 14 Surface height illustrate the value of the flux, while the colorscale indicates pointwise fitting error.

showed a $1.46 \%$ difference in $L^{2}$ norm with respect to the original MK flux (see Fig. 3)

The same derivation is carried out for all the fluxes involved in the model, resulting in the following expressions:

$$
\begin{gathered}
V_{\mathrm{PDH}}=p_{6}\left(1-p_{7} \mathrm{e}^{-p_{8}\left[\mathrm{Ca}^{2+}\right]_{\mathrm{m}}}\right)\left(p_{9}+\mathrm{e}^{F / R T \Delta E_{\mathrm{resp}}}\right)^{-1} \\
V_{\mathrm{F} 1 \mathrm{~F} 0}=p_{10} \sigma\left(\Delta G_{\mathrm{p}, \mathrm{m}}, 1,0, p_{11}, p_{12}\right) \sigma\left(\Delta p, 1,0, p_{13}, p_{14}\right) \\
V_{\mathrm{ANT}}=\frac{p_{15}\left(1-p_{16} \mathrm{e}^{F / R T\left(\Delta G_{\mathrm{p}, \mathrm{c}}-\Delta G_{\mathrm{p}, \mathrm{m}}-\Delta p\right)}\right)}{\left(1+\frac{p_{16}}{p_{17}} \mathrm{e}^{F / R T\left(\Delta G_{\mathrm{p}, \mathrm{c}}-p_{18} \Delta p\right)}\right)\left(1+p_{17} \mathrm{e}^{-F / R T \Delta G_{\mathrm{p}, \mathrm{m}}}\right)} \\
V_{\mathrm{leak}}=p_{19} \exp \left(p_{20} \Delta p\right) \\
V_{\mathrm{NaCa}}=p_{21}\left[\mathrm{Ca}^{2+}\right]_{\mathrm{m}}\left(p_{22}+\left[\mathrm{Ca}^{2+}\right]_{\mathrm{m}}\right)^{-1} \\
\max \left(0,\left[\mathrm{Ca}^{2+}\right]_{\mathrm{c}}\left(p_{23} \Delta p+p_{24}\right)+p_{25} \Delta p+p_{26}\right)
\end{gathered}
$$

The $L^{2}$ fitting error varies between $0.65 \%-5.6 \%$ for the different fluxes.

\section{Discussion, perspectives}

Together with the ODE system, our model includes a total of 6 state variables and 33 parameters. The number of equations and parameters is low enough to make very efficient computations. This allows for an extensive use of the model as needed by sensitivity analysis, and parameter calibration methods, which constitute our forthcoming work. As a first step, Sobol indices method will be used to determine the sensitivity of our flux expressions to parameters $p_{i}$, in order to reduce their number. A similar analysis will then be performed using the results of the ODE as output.

In parallel, effort will be made to reconnect the parameters as much as possible to a biophysical meaning, which is for now lacking.
Finally, we intend to add key mechanisms to our model, following a similar approach. In particular, there is evidence that reactive oxygen species (ROS) and the mitochondrial permeability transition pore ( $\mathrm{mPtP})$ have a crucial role in calcium handling, in particular in an arythmogenic context.

\section{Acknowledgments}

The authors have been partly granted by the French national agency throughout the research project MITOCARD (ANR-17-CE11-0041).

\section{References}

[1] Bertram R, Pedersen M, Luciani D, Sherman A. A simplified model for mitochondrial ATP production. J Theor Biol 2006; 243(4):575-586.

[2] Magnus G, Keizer J. Minimal model of beta-cell mitochondrial $\mathrm{Ca}^{2+}$ handling. Am J Physiol Cell $\mathrm{Ph} 1997$; 273(2):C717-C733.

[3] Gambardella J, Trimarco B, Iaccarino G, Santulli G. New insights in cardiac calcium handling and excitation-contraction coupling. Springer International Publishing, 2018; 373-385.

[4] Deschodt-Arsac V, Calmettes G, Gouspillou G, Rouland R, Thiaudiere E, Miraux S, Franconi JM, Diolez P. System analysis of the effect of various drugs on cardiac contraction energetics. Biochem Soc T 2010;38(5):1319-1321.

[5] Diolez P, Deschodt-Arsac V, Calmettes G, Gouspillou G, Arsac L, dos Santos P, Jais P, Haissaguerre M. Integrative methods for studying cardiac energetics. Springer New York, 2015; 289-303.

[6] Saa A, Siqueira K. Modeling the ATP production in mitochondria. Bulletin of Mathematical Biology 2013; 75(9):1636-1651.

[7] Cortassa S, A MA, Marbân E, Winslow R, O'Rourke B. An integrated model of cardiac mitochondrial energy metabolism and calcium dynamics. Biophys J 2003; 84(4):2734-2755.

[8] Nicholls D, Ferguson S. Bioenergetics (Third Edition). Academic Press, 2003.

[9] Hill TL. Free energy transduction in biology: the steady-state kinetic and thermodynamic formalism. Academic Press, 1977.

Address for correspondence:

Michael Leguèbe

Inria Bordeaux-Sud-Ouest

200 avenue de la Vieille Tour

33405 TALENCE CEDEX, France

michael.leguebe@inria.fr 